\title{
Adsorption and Diffusion Phenomena in Crystal Size Engineered ZIF-8 MOF
}

\author{
Shunsuke Tanaka ${ }^{\dagger \neq}$, Kosuke Fujita ${ }^{\dagger}$, Yoshikazu Miyake ${ }^{\dagger \neq}$, Manabu Miyamoto ${ }^{8}$, Yasuhisa \\ Hasegawa', Takashi Makino', Stijn Van der Perre ${ }^{\perp}$, Julien_Cousin Saint Remi $\perp^{*}$, Tom \\ Van Assche ${ }^{\perp}$, Gino V. Baron ${ }^{\perp}$, and Joeri F. M. Denayer ${ }^{\perp}$ \\ 'Department of Chemical, Energy and Environmental Engineering and fOrganization for Research \\ and Development of Innovative Science and Technology (ORDIST), Kansai University, 3-3-35 \\ Yamate-cho, Suita-shi, Osaka 564-8680, Japan \\ 'Department of Chemistry and Biomolecular Science, Gifu University, 1-1 Yanagido, Gifu 501-1193, \\ Japan \\ Research Center for Compact Chemical System, National Institute of Advanced Industrial Science \\ and Technology (AIST),4-2-1 Nigatake, Miyagino-ku, Sendai, Miyagi 983-8551, Japan \\ เDepartment of Chemical Engineering, Vnije Universiteit Brussel, Pleinlaan 2, B-1050 Brussel, \\ Belgium \\ jcousins@vub.ac.be
}

ZIF-8 is a flexible zeolitic imidazolate framework (ZIF), member of the metal-organic framework (MOF) porous materials, whose narrow pore structure swings open by reorientation of imidazolate linkers and expands at high loading of guest molecules. This nanoporous material has been identified as a potential candidate for the energy-efficient adsorptive recovery of alcohols from diluted aqueous mixtures, such as obtained with the acetone-butanol-ethanol (ABE) fermentation [1].

In this work, the crystal size dependency of both structural transitions induced by N2 and Ar adsorption was investigated, from which the results, obtained with a volumetric technique, will be presented. On the other hand, the diffusion of $n$-butanol into well-engineered ZIF-8 crystals with identical surface area and micropore volume was also explored. Results acquired with two gravimetric methods (static and dynamic versions) will be shown. It was found that the crystal downsizing of nanoporous ZIF-8 adsorbent affects its structural flexibility observed from the equilibrium adsorption and desorption data of N2 and Ar. Adsorption kinetics of n-butanol in ZIF-8 showed to be strongly influenced by the crystal size, however, not according to a classical intracrystalline diffusion mechanism. Our results suggest that the structural transitions and uptake rates are dominated by crystal surface effects. Crystal downsizing increases the importance of such surface barriers [2].

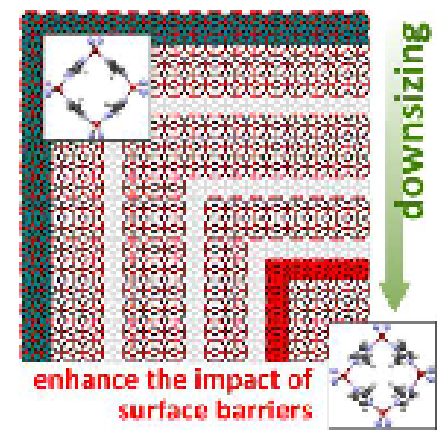

Figure 1: Schematic representation of the effect of crystal-downsizing on the enhancement of the contribution of surface barriers in the structural transition and uptake of guest-molecules by a nanoporous ZIF-8 crystal [2].

\section{References}

[1] J. Cousin Saint Remi, T. Remy, V. Van Hunskerken, S. van de Perre, T. Duerinck, M. Maes, D. De Vos, E. Gobechiya, C.E.A. Kirschhock, G.V. Baron, JF.M. Denayer. Biobutanol Separation with the MetalOrganic Framework ZIF-8 ChemSusChem 4, 1074-1077 (2011).

[2] S. Tanaka, K. Fujita, Y. Miyake, M. Miyamoto, Y. Hasegawa, T. Makino, S. Van der Perre, J. Cousin Saint Remi, T. Van Assche, G.V. Baron, J.F.M. Denayer: Adsorption and Difflusion Phenomena in Crystal Size Engineered ZIF-\& MOF J. Phys. Chem. C 119, 28430-28439 (2015). 